\title{
How to implement real-time interaction between participants in online surveys: A practical guide to SMARTRIQS
}

\author{
Andras Molnar*
}

*Department of Social and Decision Sciences, Carnegie Mellon University

5000 Forbes Ave, Pittsburgh, PA 15213, USA

E-mail: andrasm@andrew.cmu.edu

Last updated: February 1, 2020

\begin{abstract}
SMARTRIQS is a free and open-source application that fills in a crucial gap in contemporary experimental research methods: It offers researchers the ability to design online surveys that feature real-time interaction between participantsincluding live text chat - without requiring researchers to learn any programming language, install any software, or pay for any third-party services. This paper not only provides an overview of SMARTRIQS and its potential applications but also walks readers through the step-by-step instructions for setting up a particular study (Dictator Game with chat). These instructions start from the very basics, assuming no prior expertise in online experimentation, and are accessible to everyone, even those who are less - or not at all—familiar with Qualtrics.
\end{abstract}

\section{KEYWORDS}

chat | online experiment | Qualtrics | real-time interaction | survey design 


\section{INTRODUCTION}

Online experimentation is becoming increasingly popular in social sciences (Arechar, Gächter, \& Molleman, 2018; Gosling \& Mason, 2015) - largely due to the massive technological improvements in survey software (e.g., Qualtrics, 2020; SurveyMonkey, 2020), and the advance of large-scale online participant panels (Chandler, Rosenzweig, Moss, Robinson, \& Litman, 2019) and crowdsourcing platforms such as Amazon Mechanical Turk (Bohannon, 2016; Mason \& Suri, 2012; Paolacci \& Chandler, 2014), Prolific, or CrowdFlower (Peer, Brandimarte, Samat, \& Acquisti, 2017). These tools allow researchers to collect and analyze data at an unprecedented scale, speed, and efficiency, which makes them superior to conventional lab studies ${ }^{1}$ in many ways.

While lab studies typically provide researchers more experimental control over the procedures and have lower attrition rates than online experiments (Arechar et al., 2018; Zhou \& Fishbach, 2016), they have several major limitations (see "Offline RTI" in Table 1). Offline studies are usually more expensive - both in terms of participant compensation and administrative costs - require a physical lab and equipment, and have considerably smaller and less heterogeneous subject pools (Berinsky, Huber, \& Lenz, 2012; Henrich, Heine, \& Norenzayan, 2010). Also, since the number of participants who can concurrently participate in lab studies is limited, data collection takes significantly longer.

Online studies can overcome most of the limitations of conventional lab studies, but they also pose unique methodological challenges. Most importantly, implementing real-time interaction and communication in an online experiment is notoriously difficult, unlike allowing participants to seamlessly interact with each other in a conventional lab environment. Setting up such a study takes a considerable amount of time, and requires researchers either to have advanced programming skills, or to pay for third-party services and products. These challenges prevent many social scientists from utilizing online research methods in their research. Researchers who wish to study social interaction, group dynamics, communication, or any other form of interpersonal decision-making, often have to resort to simpler but also inferior alternatives, which only allow for non-real-time interaction or simulated interaction between participants (see "Online non-RTI" in Table 1).

- See Table 1 on the next page -

\footnotetext{
${ }^{1}$ This category includes both pen-and-paper experiments and computerized interactions implemented on local networks, e.g., via z-Tree (Fischbacher, 2007).
} 
Table 1. A comparison of methods for studying social interaction between participants

\begin{tabular}{|c|c|c|c|}
\hline Type & Method & Main advantages & Limitations \\
\hline (1) Offline RTI* & $\begin{array}{l}\text { Lab (e.g., z-Tree, } \\
\text { pen-and-paper studies) }\end{array}$ & $\begin{array}{l}\text { High experimental control } \\
\text { Low attrition rates }\end{array}$ & $\begin{array}{l}\text { Requires lab \& equipment; } \\
\text { Expensive; time consuming; } \\
\text { Limited subject pool }\end{array}$ \\
\hline \multirow[t]{3}{*}{ (2) Online non-RTI* } & "Wave" recruitment & Does not require programming & $\begin{array}{l}\text { Potential selection confound; } \\
\text { Slow; manual matching }\end{array}$ \\
\hline & Strategy method & $\begin{array}{l}\text { Does not require programming; } \\
\text { More observations per subject }\end{array}$ & $\begin{array}{l}\text { Might affect behavior; } \\
\text { Manual matching }\end{array}$ \\
\hline & Deception & $\begin{array}{l}\text { Does not require programming; } \\
\text { Easy setup }\end{array}$ & $\begin{array}{l}\text { Subject pool contamination; } \\
\text { Potential IRB issues }\end{array}$ \\
\hline \multirow[t]{4}{*}{ (3) Online $\mathrm{RTI}^{*}$} & $\begin{array}{l}\text { Experimental platforms } \\
\text { (e.g., oTree) }\end{array}$ & $\begin{array}{l}\text { Highly customizable; } \\
\text { Repeated, complex interaction }\end{array}$ & Requires programming \\
\hline & $\begin{array}{l}\text { Third-party services } \\
\text { (e.g. iDecisionGames) }\end{array}$ & $\begin{array}{l}\text { Highly customizable; } \\
\text { Advanced features (e.g., video chat) }\end{array}$ & $\begin{array}{l}\text { Expensive; closed source; } \\
\text { Limited experimental control }\end{array}$ \\
\hline & $\begin{array}{l}\text { Specilaized applications } \\
\text { (e.g., Chatplat) }\end{array}$ & $\begin{array}{l}\text { Does not require programming; } \\
\text { Easy setup; platform-independent }\end{array}$ & Limited to one type of interaction \\
\hline & SMARTRIQS & $\begin{array}{l}\text { Does not require programming; } \\
\text { Easy setup; highly customizable }\end{array}$ & $\begin{array}{l}\text { None of the above } \\
\text { (see Table } 2 \text { ) }\end{array}$ \\
\hline
\end{tabular}

*Note: "RTI" indicates true (non-deceptive) real-time interaction between participants. 


\section{Simple, but flawed alternatives to real-time interaction in online studies}

There are three simple methods that allow researchers to study social interactions in online experiments, without requiring participants to interact in real-time: "wave" recruitment, the strategy method, and deception. While all of these alternatives help researchers to overcome the limitations of conventional lab studies, they also introduce new limitations, which, depending on the research objectives and the standards of the researcher's discipline, can render these methods unsuitable for conducting online experiments.

Wave recruitment refers to the practice of recruiting a group of participants, recording their decisions, and displaying these decisions to a second group of participants, who are recruited in subsequent sessions. Although this alternative does not require any programming skills and is relatively easy to set up, researchers have to match participants and transmit their responses manually, which is not only very labor-intensive and error-prone, but also makes complex interactions (e.g. multiple rounds, large groups) extremely challenging - if not impossible - to implement.

Strategy method is the second alternative. When using the strategy method, participants make conditional decisions for each possible action of other participants. Similarly to the wave recruitment method, participants are "matched" post hoc, and decisions are implemented only after the experiment. While the strategy method allows researchers to collect more data per participant, it is also very labor-intensive and error-prone, since it requires the experimenter to manually match participants and determine outcomes. More importantly, participants might behave differently when their choices and preferences are elicitied using the strategy method, as opposed to a directresponse method, which might affect the validity of the experiment. For instance, Casari and Cason (2009) found that people showed significantly lower levels of trustworthiness when using the strategy method, compared to the direct-response method, which suggests that the strategy method should be used with caution when studying trust-related behaviors.

Deception is arguably the most problematic alternative to implementing real-time interaction in online studies. When using deception, participants are explicitly told or led to believe that they are interacting with other people, while they are actually "interacting" with the computer. This not only violates the research standards of several disciplines (e.g., experimental economics and experimental finance), but also conflicts with most Institutional Review Board (IRB) policies, which explicitly specify that deception can only be used when there are no reasonably effective alternative methods available to achieve the goals of the research. The practice of deceiving subjects out of convenience, because it is difficult (but not impossible) to implement real-time interaction online, is thus violating IRB guidelines. Furthermore, the excessive use of participant deception can contaminate subject pools - i.e., erode the credibility of experimental instructions and alter participants' behavior over time - especially in large-scale crowdsourcing platforms such as MTurk or Prolific (for a detailed discussion and a review of empirical evidence on the effects of deception, see Hertwig \& Ortmann, 2008). 


\section{Existing methods that allow for real-time interaction in online studies}

The existing solutions that allow for conducting interactive studies online, can be classified into three broad categories: standalone experimental platforms, closed-source third-party services, and specialized applications (see "Online RTI" in Table 1).

The first category, standalone experimental platforms such as oTree (Chen, Schonger, \& Wickens, 2016) ${ }^{2}$, offer researchers great flexibility in study design and are typically freely accessible and open-source. However, all of these platforms require users to have at least some expertise in a programming language (e.g., Python, JavaScript, PHP). Expecting all social scientists who wish to study social behavior in online settings to be accomplished programmers is akin to expecting everyone who drives a car to be a certified mechanic. Although a nice idea, it isn't practical — our limited resources can be put to better use.

Another alternative is to outsource programming tasks to a third-party or to hire a professional programmer, however, doing so can be prohibitively expensive, especially for smaller labs or junior researchers who have limited funds. Furthermore, individual programmers usually lack the experience with behavioral experimental research and need a lot of guidance and consultation when designing and editing studies. By contrast, there are private companies that specialize in designing and conducting online experiments (e.g., iDecisionGames, https://idecisiongames.com). While these services offers a great flexibility in experimental design and allow researchers to use advanced features such as live video chat between participants, researchers have no direct control over the design process and have to communicate every minor edit to the third-party company, which can substantially slow down the design and testing phase. Furthermore, since these are for-profit companies, their products are closed-source, which forces researchers to keep paying for services, even if they just want to run an exact replication of an experiment conducted by another lab. Since replicability and transparent research practices are increasingly important in the social sciences (see e.g., Collaboration*, 2015), this is a rather serious limitation.

Finally, there are specialized applications such as Chatplat (https://www.chatplat.com), that address the limitations of both generic experimental platforms and third-party services. These applications are free, do not involve any programming, and can be set up relatively easily. However, these advantages come at the expense of flexibility: Specialized applications are designed for a specific type of interaction, and their applicability is limited to a narrow range of experiments. For example, Chatplat allows participants to chat with each other in online surveys, but does not allow for setting up different types of studies (e.g., which require transmitting decisions).

\footnotetext{
${ }^{2}$ Other popular platforms: ConG (Pettit, Friedman, Kephart, \& Oprea, 2014), LIONESS Lab (Giamattei, Molleman, Yahosseini, \& Gächter, 2019), MWERT (Hawkins, 2015), nodeGame (Balietti, 2017), Psynteract (Henninger, Kieslich, \& Hilbig, 2017), SoPHIE (Hendriks, 2012), and TurkServer (Mao, Chen, Parkes, \& Procaccia, 2012).
} 


\section{A new tool for studying real-time interaction in online studies: SMARTRIQS}

The previous sections highlight not only the demand for, but also the limitations of, methods that allow researchers to implement real-time interaction between participants in online studies. These methods are either too difficult to use (i.e., require programming), too expensive, or have limited applications. SMARTRIQS is a first-of-its-kind solution that addresses all of these challenges, and allows researchers to conduct interactive experiments without the hassle of programming or paying for extra services. Table 2 summarizes the main features and limitations of SMARTRIQS:

Table 2. Features and limitations of SMARTRIQS

\begin{tabular}{|c|c|c|}
\hline Feature & Description & Limitation \\
\hline Cost and access & Free and open-source & Requires Qualtrics account \\
\hline Implementation & Default or custom (private) server & - \\
\hline Ease of use & $\begin{array}{l}\text { Requires no programming; } \\
\text { Requires no installation }\end{array}$ & - \\
\hline Integation with Qualtrics & All data saved in Qualtrics & - \\
\hline Participant matching & Fixed groups & No re-matching \\
\hline Group size & $\begin{array}{l}2-8 \text { participants per group; } \\
\text { Unlimited number of groups }\end{array}$ & Max. 8 participants per group \\
\hline Randomization & $\begin{array}{l}\text { Random or custom assignment } \\
\text { to roles and conditions }\end{array}$ & - \\
\hline Type of interaction & $\begin{array}{l}\text { One-shot or repeated; } \\
\text { synchronous or sequential }\end{array}$ & - \\
\hline $\begin{array}{l}\text { Types of data that } \\
\text { can be transmitted }\end{array}$ & $\begin{array}{l}\text { Any data type that is supported } \\
\text { in Qualtrics (numeric, text, scale) }\end{array}$ & $\begin{array}{l}\text { Transmission of personal data } \\
\text { may be prohibited (consult IRB) }\end{array}$ \\
\hline Supported communication & $\begin{array}{l}\text { Highly customizeable text chat } \\
\text { (e.g., group or private chat; } \\
\text { multiple stages; custom format) }\end{array}$ & No audio or video chat \\
\hline \multirow[t]{6}{*}{ Advanced features } & Waiting rooms; & \\
\hline & $\begin{array}{l}\text { Built-in math operations } \\
\text { (e.g., sum, rank, min, max); }\end{array}$ & \\
\hline & Dropout management; & \\
\hline & Bots and default responses; & \\
\hline & Free survey templates and demos; & \\
\hline & Data collection monitor & \\
\hline
\end{tabular}


The conceptual framework and the architecture of SMARTRIQS were introduced in Molnar (2019). However, that paper was addressed to a specialist audience (researchers in experimental finance) and did not provide any instructions to how to design interactive studies. The current paper, by contrast, focuses on the practical applications of SMARTRIQS and provides researchers detailed, step-by-step instructions and hands-on guidance. The guide is designed to help researchers to become familiar with SMARTRIQS and to set up and customize interactive studies effortlessly, without requiring them to have any prior experience with online experimentation. The paper also lists and discusses various applications across several disciplines of the social sciences.

\section{STEP-BY-STEP TUTORIAL}

This section provides a comprehensive guide to using SMARTRIQS and walks the reader through the step-by-step instructions for setting up a particular study: the Dictator Game. In this classic dyadic interaction, participants are randomly assigned to either the role of the "Allocator" or the "Recipient." At the beginning of the experiment the Allocator is endowed with an amount (e.g., 100 tokens). Then, she can transfer any amount out of her endowment to the Recipient. The Recipient does not make any decision, simply receives whatever amount is transferred to her. Variations of this experiment are widely used to measure social preferences and attitudes towards others (Forsythe, Horowitz, Savin, \& Sefton, 1994). To demonstrate the live chat feature of SMARTRIQS, this section also covers how to allow chat between participants.

\section{Prerequisites}

Researchers need the following before they start designing interactive studies with SMARTRIQS:

1. An institutional subscription to the Qualtrics Survey Software. Free or trial accounts are not supported. This tutorial does not assume any prior experience with Qualtrics. However, having some familiarity with certain Qualtrics concepts (e.g., Survey Flow, Embedded Data, Branch Logic, Piped Text) is recommended, as it will make it easier to follow this guide and to understand how SMARTRIQS works. For these who are new to Qualtrics or wish to refresh their knowledge, there are plenty of free tutorials available online. For example, Dare McNamara has two excellent video tutorials: Beginner Qualtrics Training (10 minutes) and Advanced Qualtrics Training (17 minutes).

2. A SMARTRIQS researcher ID, which can be obtained by submitting the registration form. Researchers have to provide their name and email address, and accept the Data Policy, in order to receive their unique researcher ID.

3. One or more SMARTRIQS survey templates. These "Qualtrics Survey Format" (QSF) files can be downloaded from the OSF repository. 


\section{Importing survey templates (QSF files) to Qualtrics}

Throughout this tutorial we will use the "Generic Interactive Survey Template" (GIST). However, researchers can start with any template that works best for them. The GIST has every SMARTRIQS feature (including chat) and using it is recommended for custom studies that are very different from other templates. After logging in to Qualtrics, click on "Create new project" (top right corner of the main page), then select "Survey" under "Create your own" and click on "From a File / Choose file." In the pop-up window, select the QSF file you wish to import ("Generic_Interactive_Survey_Template_GIST.qsf" in this tutorial) and click "Open." Finally, rename the project and click on "Get Started."

\section{Matching participants: the MATCH block}

Before participants can chat or interact with each other, they have to be matched first. In SMARTRIQS surveys this happens in the "MATCH block." SMARTRIQS offers a lot of flexibility in customizing interactions (e.g., group size, number of stages, participant roles). Note that most of these settings must be set before the MATCH block. To set up a MATCH block, open the imported survey and then open the Survey Flow (top left of the main page). On the top of the Survey Flow there are two green panels titled "Required parameters" and "Optional parameters" (see Figure 1).

These panels contain the Embedded Data fields (olive green), which are essentially the variables and parameters used in Qualtrics survey. SMARTRIQS studies use Embedded Data for two purposes: 1) as parameters that define the characteristics of the interaction, and 2) as variables that store participants' responses. Throughout the subsequent sections of paper, boldface text indicates Embedded Data fields. After scrolling down in the Survey Flow, there will be several grey panels and additional green panels with more Embedded Data. Each grey panel represents a Question Block. Blocks are either built-in SMARTRIQS blocks that are responsible for various types of interaction (MATCH, CHAT, SEND, GET, and COMPLETE) or standard Qualtrics blocks that contain regular survey items (e.g., instructions, decisions, questionnaires). The latter can be added and edited manually in the Survey Editor. The first panel of Embedded Data contains the required parameters for matching participants. These parameters must be set before the MATCH block:

- researcherID: Enter the SMARTRIQS researcher ID here.

- studyID: Enter the name of the study here. The name can be any combination of alphanumeric characters (0-9, A-Z, a-Z), dash (-) or underscore (-), up to a length of 256 characters. For example: Dictator_Game_Demo-1. Note that names are case-sensitive.

- groupID: This is the field that identifies groups within a study. Leave this field blank, this will be automatically populated by SMARTRIQS.

- participantID: Keep the default value in this field (\$e:/Field/ResponseID) in order to assign the built-in Qualtrics response ID to participants (recommended). This guarantees that everyone will receive a random, unique, and anonymous ID. 


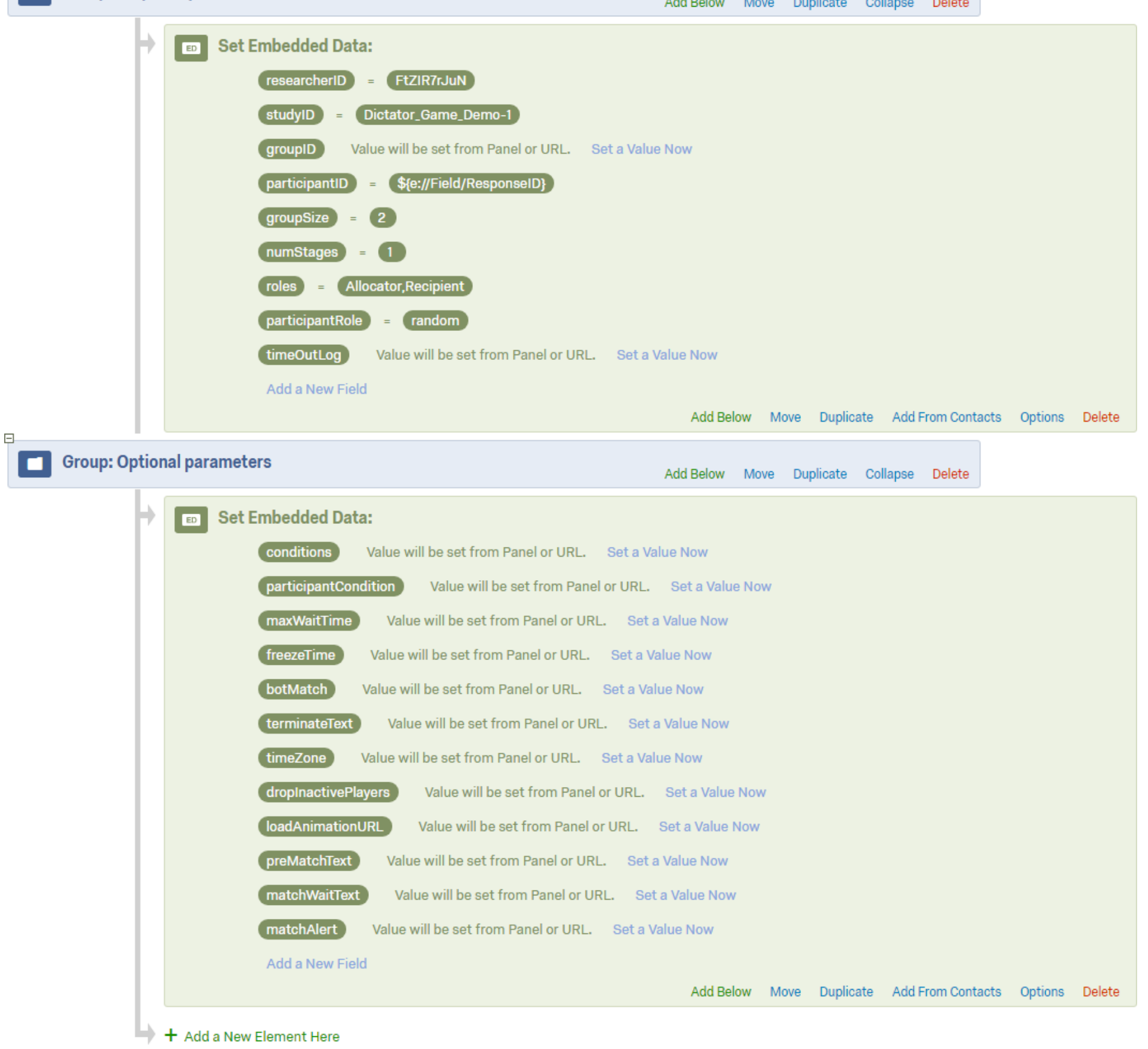

Show Block: MATCH BLOCK (4 Questions)

Add Below Move Duplicate Delete

Figure 1. Sample screenshot of the setup of required and optional parameters before the MATCH block (Dictator Game). Note that both the "Required parameters" and "Optional parameters" panels must be placed before (above) the MATCH block in the Survey Flow. 
- groupSize: Determine how many participants should be in each group. SMARTRIQS supports group interaction up to 8 participants per group. Set this to 2 for dyadic interactions.

- numStages: Determine the number of decisions to be transmitted across participants. This usually corresponds to experimental stages. If participants take turns or make multiple decisions, there should be multiple stages. In the Dictator Game example, we set this field to 1, since there is only one decision that needs to be transmitted (the Allocator's transfer).

- roles: Determine the set of available roles within groups, where the roles are separated by commas. For example: Allocator,Recipient. Note that roles are case-sensitive, and that there is no space between the comma and the roles.

- participantRole: Determine the role of the participant. To assign roles randomly within groups, enter random here. To learn more about custom role assignment methods, visit https://smartriqs.com/randomization.

- timeOutLog: SMARTRIQS saves error logs in this field, for example, when participants drop out from the study. Leave this field blank, this will be automatically populated.

The second panel contains optional parameters, which allow researchers to customize the nonessential features of the MATCH block (e.g., messages displayed before and during matching). In this tutorial we leave these fields blank, and let SMARTRIQS apply the default values. To learn more about optional parameters, visit https://smartriqs.com/matching/\#matchBlock.

\section{Real-time communication between participants: the CHAT block}

One of the most advanced features of SMARTRIQS is its built-in chat interface, which allows two or more participants to chat in real-time. The chat feature is flexible and customizable: Researchers can set up chat with or without a time limit; allow chat between the whole group or just some participants within the group; have multiple stages of chat within the study (e.g., interrupt the chat with a task, then continue the chat after participants finish the task); or customize the design of the chat window (e.g., time stamps, window size, chat instructions).

SMARTRIQS saves the content of the CHAT block, including participants' roles, messages, and time stamps (if set) to an Embedded Data field (chat log) that can be accessed by downloading the collected data. The CHAT block has only one required parameter: chatName, which refers to the name of this chat log. First, we set up an Embedded Data field that will serve as the chat $\log$. The name of this field can be any combination of alphanumeric characters $(0-9, \mathrm{~A}-\mathrm{Z}, \mathrm{a}-\mathrm{Z})$, dash (-) or underscore (_), up to 32 characters length. Note that the name is case-sensitive. In this example we keep the default name of this field (chatLog). The value of this field should always be set to text. Then, we define the chatName parameter. The value of this parameter should be the name of the chat log field above, which is chatLog in this example (Figure 2). 
ED Set Embedded Data:

chatLog $=$ text

Add a New Field

Add Below Move Duplicate Add From Contacts Options Delete

ED Set Embedded Data:

chatName $=$ chatLog

Add a New Field

Add Below Move Duplicate Add From Contacts Options Delete

ED Set Embedded Data:

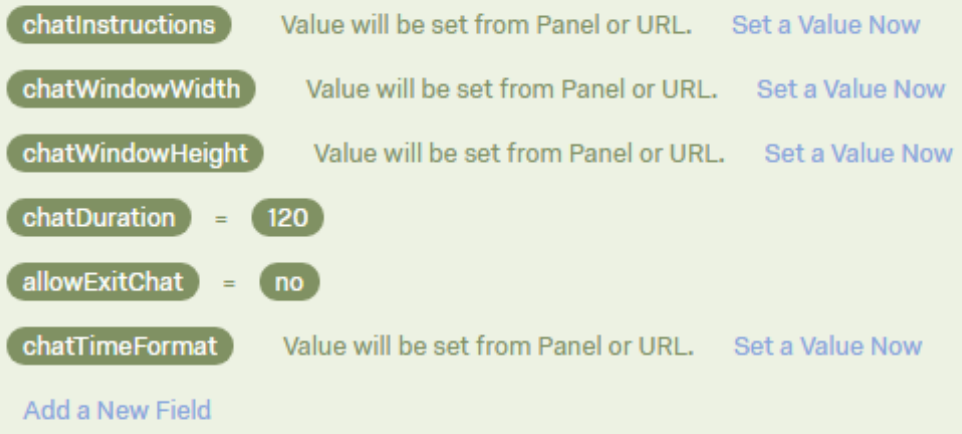

Add Below Move Duplicate Add From Contacts Options Delete

Show Block: CHAT BLOCK (2 Questions)

Add Below Move Duplicate Delete

Figure 2. Sample screenshot of the setup of the CHAT block. The chat log is saved in the first Embedded Data field (chatLog). The second field (chatName) is the required parameter for the CHAT block, and should always refer to the name of the chat log defined above. The third green panel contains the optional parameters for the CHAT block. Embedded Data fields should always be placed before (above) the CHAT block in the Survey Flow.

The CHAT block has six optional parameters, which allow researchers to apply time limits and customize the chat design. In this example we keep the default design but implement a time limit of two minutes by setting the chatDuration parameter to 120 (seconds). This means that the chat will end after two minutes. By default - if this field is left blank - there is no time limit, and participants can chat for as long as they wish. We also set the allowExitChat parameter to no, which indicates that participants are not allowed to exit the chat before the time is up (i.e., they must chat for two minutes). By default - if this field is left blank-participants can exit any time.

To learn more about time limits, custom designs, and more advanced chat features (i.e., multiple stages, interrupted chat, private and group chat), visit https://smartriqs.com/chat. Researchers who do not want to include any chat in their studies, should delete the CHAT block from the Survey Flow, along with the Embedded Data fields above the CHAT block. 


\section{Adding instructions and recording responses: Question Blocks}

At this point, participants can already chat with each other. If the study does not require any other response to be transmitted, the SEND and GET blocks should be deleted from the Survey Flow. In that case, the survey is ready for testing and data collection. However, if the study requires responses to be transmitted (e.g., the Allocator's transfer), researchers should add new Question Block(s). In this example, we add a block that allows the Allocator to make a decision (see Figure S1 in the Appendix):

1. Close the Survey Flow, scroll down to the bottom of the CHAT block in the Survey Editor and click on "Add Block." This adds a blank Question Block to the survey.

2. Rename the new block by clicking on its name (e.g., "Allocator's Transfer").

3. Click on "Create a New Question."

4. Click on the green button labeled "Multiple choice" on the right side of the screen (below "Change Question Type") and select "Slider."

5. Change "Choices" to 1.

6. Check "Force Response" to prevent participants from proceeding without making a decision. It is best practice to always force responses in interactive studies.

7. Label the question (e.g., "Allocator's Transfer") and the choice ("Your transfer"), and add some instructions by editing the question text ("You have been assigned to the role of the Allocator. Please indicate below how many tokens you want to transfer to the Recipient").

Next, we repeat the above procedure for the Recipient. In the Dictator Game the Recipient does not make any decisions, she simply receives the Allocator's transfer. However, we still need to add another Question Block, in which we inform the Recipient about the transfer. Add a new Question Block and rename it to "Recipient's Payment." Create a new question, and change the question type to "Descriptive Text." Label the question (e.g., "Recipient's Payment") and add

the following text: "The Allocator has decided to transfer you $\$\{$ e://Field/transfer $\}$ tokens" (see Figure S2 in the Appendix).

The expression following the $\$$ sign is a Qualtrics Piped Text, which represents a dynamic text that depends on a variable or a previous response. When participants take the survey, they see the actual value of this variable (e.g., 50), instead of the expression. In this example, this Piped Text depends on an Embedded Data field called transfer, which has not been defined yet. We will add this field to the Survey Flow when we set up the GET block. 


\section{Setting up role-dependent questions and blocks: Branch Logic}

In most studies participants are presented different instructions and make different decisions, depending on their roles. In the Dictator Game the Allocator transfers an amount, while the Recipient does not make any decision. This means that we should not display every block to everyone. Instead, we should present the "Allocator's Transfer" block to Allocators only, and the "Recipient's Payment" block to Recipients only. To achieve this, we utilize the Qualtrics feature called Branch logic, which allows for displaying only certain blocks to participants, conditioned on some criteria.

Open the Survey Flow, and scroll down to the section with the two new blocks created in the previous section. Click on "Add Below" on the panel of the CHAT block and select "Branch." Then click on "Add a Condition," and select "Embedded Data" from the drop-down menu. Enter participantRole in the first empty field, and enter Allocator in the second empty field. Note that these are case-sensitive. Then click on "Move" on the panel of the "Allocator's Transfer" block, and while holding the left mouse button, drag this block under the branch. Repeat this process for the Recipient: Add a new branch below the CHAT block, add a condition, and select "Embedded Data." Enter participantRole in the first empty field and Recipient in the second empty field. Finally, drag the "Recipient's Payment" block under this new branch (see Figure S3 in the Appendix).

The Dictator Game is an example with a single decision and "one-shot" interaction, so we do not have to add more questions. However, one of SMARTRIQS' greatest strengths is the ability to facilitate multi-stage interactions. For example, researchers might want to have participants to make consecutive decisions. For practical examples, see the demo studies with more complex Branch Logic (e.g., Trust Game, Ultimatum Game, or Third-Party Punishment) at https://smartriqs.com/demos. The corresponding QSF templates can be downloaded from the OSF repository.

\section{Transmitting responses across surveys: the SEND and GET blocks}

So far we have added all the necessary questions to the survey, however, these are still individual responses, which need to be transmitted across participants. SMARTRIQS uses two blocks to achieve this: the SEND block submits responses to the SMARTRIQS server, while the GET block retrieves responses from the server. In the Dictator Game, we want to send the Allocator's transfer to the server, so that the Recipient can retrieve it.

Sending data: the SEND block. Open the Survey Flow and scroll down to the SEND block. There are two green panels above the SEND block: The top panel has one Embedded Data field (response), while the bottom one has two fields (sendData and sendStage). All three are required parameters, and they should always be set before (above) the SEND block. There are no optional parameters for the SEND block. The green panels and the SEND block should always be placed after (below) the question block that contains the response to be transmitted. In this example, the response to be transmitted is in the "Allocator's Transfer" block, so move the two green panels and the SEND block under the Allocator's branch and below the "Allocator's Transfer" block (Figure 3). The next step is setting up the values of the three required Embedded Data fields. By default, the name of the first field is response but it is best practice to change this to something 
If participantRole is Equal to Allocator Edit Condition

Move Duplicate Options Collapse Delete

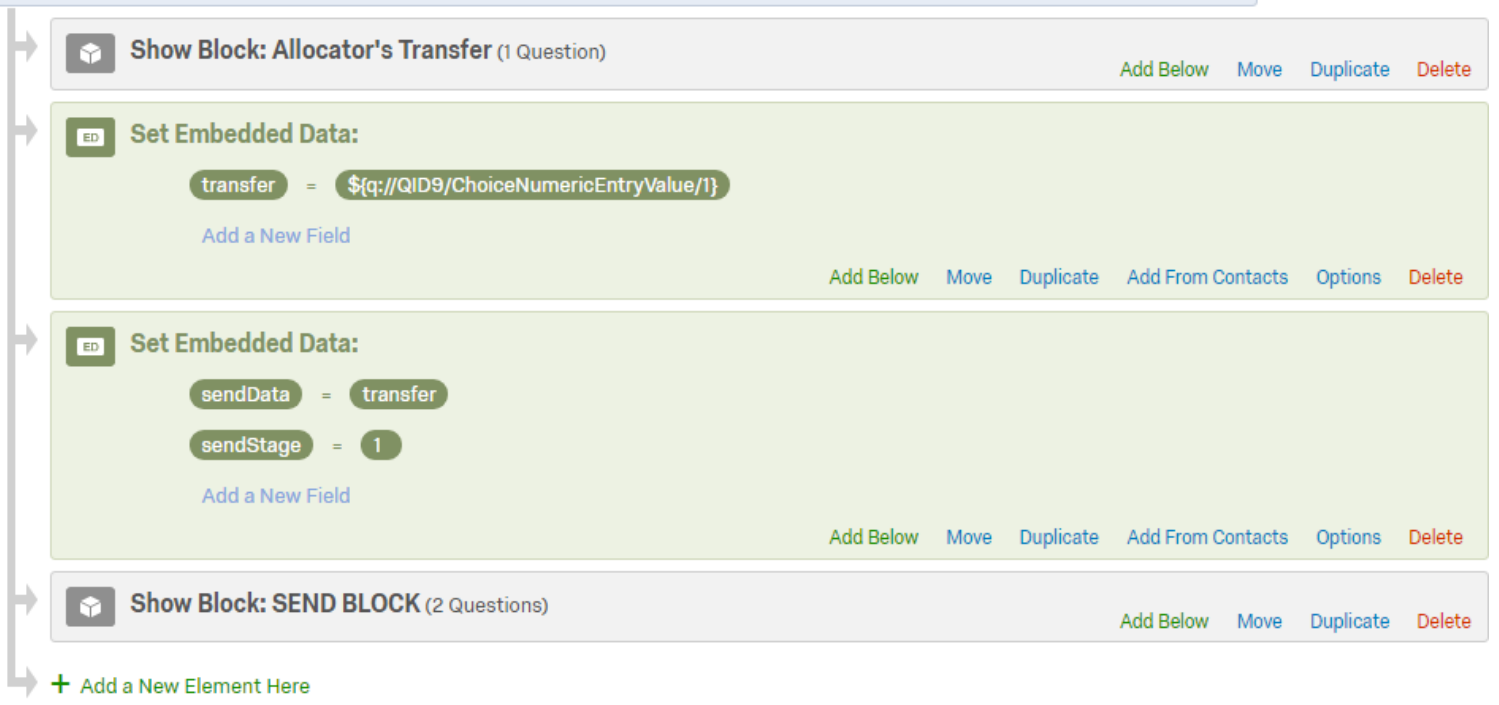

Figure 3. Sample screenshot of the setup of the SEND block (Dictator Game).

more informative and specific (max. 32 alphanumeric characters: 0-9, A-Z, a-z, -, _). Not changing the name can not only make data analysis difficult but also lead to errors in the Survey Flow. In this example, we rename this field to transfer. The value of this field should always be a Piped Text that refers to a particular response provided by the participant. This response can be of any type: multiple choice, scale, open-ended text, etc. In this example, the Piped Text should refer to the Allocator's decision. Click on "Set a Value Now," then click on the blue arrow, and select "Insert Piped Text $\rightarrow$ Survey Question." The pop-up menu will show all the eligible questions and other variables than can be inserted as Piped Text. Find the question that has the response to be transmitted (in this example: "Allocator's Transfer"). When this question is highlighted, click on the response to be transmitted ("Your Transfer," see Figure S4 in the Appendix). Note that "Your Transfer" is the manually set label of the response, not the response itself. It is worth reiterating that the use of intuitive and informative labels is best practice. In this instance, it will make it easier to insert the correct response using Piped Text.

The second field (sendData) indicates which previously defined Embedded Data field should be sent to the server. The value of this field should always be the name of another Embedded Data field - it should not refer directly to a question response. In this example, change the value of sendData to transfer. This indicates that the SEND block will access the value stored in the transfer field, and send that value to the SMARTRIQS server. The third field (sendStage) specifies which experimental stage does the selected response correspond to. The value of this field should be a positive integer that is less than or equal to the numStages parameter, which was defined before the MATCH block. Since the Dictator Game has only one stage (numStages = 1), set sendStage $=1$. 
Retrieving data: the GET block. The function of the GET block is to retrieve responses from the SMARTRIQS server. A response cannot be retrieved if it was not previously transmitted to the server via a SEND block. In this example, the GET block retrieves the Allocator's transfer from the server and saves this response to the Recipient's survey. Qualtrics can then display the response to the Recipient. Open the Survey Flow and scroll down to the GET block. There are two green panels above the SEND block: the top one has three required fields of Embedded Data (getData, getStage, and saveData), while the bottom one has nine optional fields. The green panels should always be placed before (above) the GET block. Always place the GET block before (above) the question block(s) in which the retrieved response is displayed. Move the panels and the GET block under the Recipient branch, but above the "Recipient's Payment" block (Figure 4).

\section{$\checkmark$ Then Branch If:}

If participantRole Is Equal to Recipient Edit Condition

\section{Move Duplicate Options Collapse Delete}

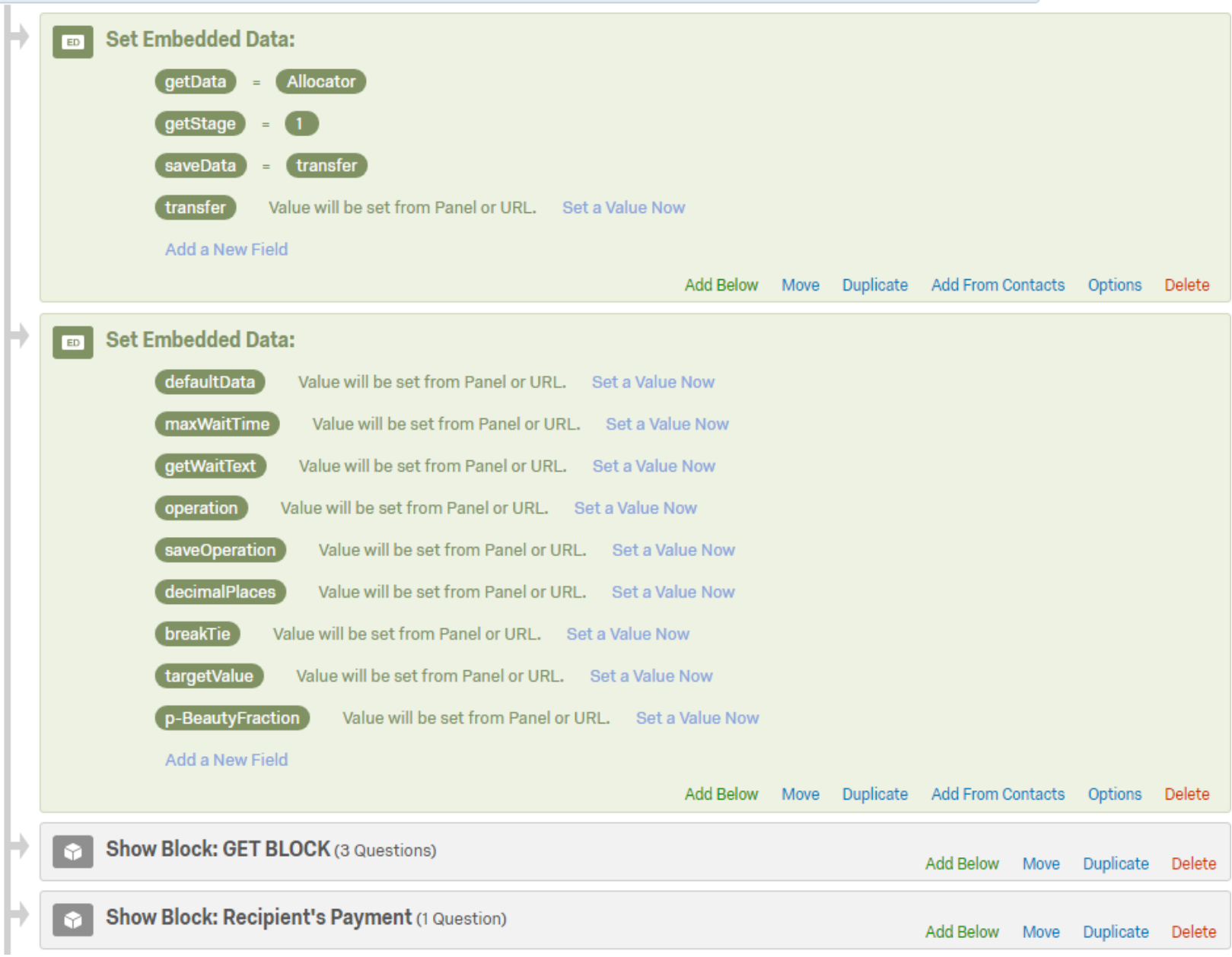

Figure 4. Sample screenshot of the setup of the GET block: required parameters (top green panel) and optional parameters (bottom green panel). These panels and the GET block should always be placed before (above) the block in which the retrieved response is displayed ("Recipient's Payment" in this example). 
The getData field specifies which response should be retrieved from the server. The value of this field should be the role of the participant whose response is retrieved (e.g., Allocator). The getStage field specifies which stage the response is retrieved from. This should be a positive integer that is less than or equal to the numStages parameter. Since the Allocator's response is transmitted in Stage 1, set getStage $=1$. The saveData field specifies the name of the Embedded Data field into which the retrieved response is saved. This Embedded Data field should be added manually to the Survey Flow. To do this, click on "Add a New Field," and name the new field, leaving its value blank. Then use the name of this new field as the value for saveData. For example, create a new field named transfer, and set the value of saveData to transfer.

The GET block has nine optional parameters. The defaultData parameter specifies a default (computer-generated) response, which is applied if the original (human) response cannot be retrieved. This occurs in two cases:

\section{If the other "participant" is a bot. ${ }^{3}$}

2. If the other participant has "timed out," that is, if he or she has not submitted a response before the maximum waiting time expired (see maxWaitTime).

By default, there are no bots or default responses in SMARTRIQS surveys. To learn more about using bots and default responses, visit https://smartriqs.com/bots.

The maxWaitTime parameter specifies the maximum waiting time limit (in seconds). Participants will wait in the GET block until the response is successfully retrieved, or until they reach this time limit. If they reach the time limit before retrieving a response, the survey is either terminated (the default setting), or a default (computer-generated) response is applied (if using bots or using default responses is allowed). If this optional parameter is left blank, participants will wait up to 3 minutes for each response. The getWaitText parameter customizes the message displayed to participants while they are waiting in the GET block, while the other six optional parameters allow researchers to do mathematical operations on retrieved responses (for example, to calculate the average or the maximum of retrieved responses). In the Dictator Game example there is no need for these optional settings or mathematical operations, so leave these fields blank. To learn more about optional parameters and operations, visit https://smartriqs.com/sending-retrieving/\#getBlock.

\section{Concluding the study: the COMPLETE block}

The COMPLETE block concludes the survey and indicates that the participant has completed the study. It also records issues in the timeOutLog field (e.g., participant dropouts and response timeouts). If a participant has not experienced any issue during the study, the timeOutLog field will take the following value: "OK - no issues." Otherwise, the field will contain a brief description of the issue encountered (e.g., "Allocator timed out in stage 1").

\footnotetext{
${ }^{3}$ Note that to avoid any deception, participants in SMARTRIQS are always informed in real-time whether they have been matched with other participants or bots. This is a built-in feature that cannot be customized.
} 
The COMPLETE block does not require any parameters and cannot be customized, so there is no need to define any Embedded Data fields before this block. Do not put any blocks after the COMPLETE block, otherwise SMARTRIQS could incorrectly indicate that a participant has completed the study, even if that participant actually dropped out at some point after the COMPLETE block. The only exception is the End of Survey Element which should always be placed after the COMPLETE block. This element is optional: Researchers can include it to display a custom end of survey message or to redirect participants to another page (Figure S5 in the Appendix).

\section{Launching studies and monitoring data collection}

To launch the study, save and exit the Survey Flow, then click on the green "Publish" button (top right), and click "Publish" again. Qualtrics will generate a public URL address ("Anonymous Survey Link"), which gives access to the survey. If the study has been already published, the URL can be accessed under "Distributions $\rightarrow$ Anonymous Link." Thoroughly test any survey and ensure that all features work as expected before distributing the study link to participants. To test the Dictator Game, open the link in two tabs (or on two devices), and complete the study in both roles. If everything has been set up properly, the study should conclude without any issues, and the data should be available under the Data \& Analysis menu in Qualtrics. Otherwise, an error message will be displayed, describing the issue. To optimize participant experience, and to minimize the risk of having technical issues, please read the best practices and other useful tips for data collection at https://smartriqs.com/best-practices.

SMARTRIQS has a "progress monitor" website that allows researchers to monitor data collection and participant activity. In addition to displaying the status of each group, the progress monitor also shows the activity and responses of individual participants in real-time (see Figure 5 in the Appendix). To access the progress monitor, go to https://server.smartriqs.com/php/monitor.html and enter the researcherID and studyID.

\section{SERVER TIME: Sep/06/2019 1:09:08 MONITORING ACTIVE}

\section{StudyID = Dictator_Game_Demo-1}

\begin{tabular}{|c|c|c|c|c|c|c|c|c|}
\hline \multicolumn{9}{|c|}{ Generate csv file } \\
\hline Group ID & Condition & Group status & Allocator & Last active & Allocator\#1 & Recipient & Last active & Recipient\#1 \\
\hline 8 & null & MATCHING... & R_1r7acBSXoy... & 09/06 1:09:08 & {$[\ldots .]$.} & [open] & {$[\ldots .]$.} & {$[\ldots .]$.} \\
\hline 7 & null & IN PROGRESS: $0 \%$ & R_3PnJdRyFrP... & 09/06 1:08:44 & {$[\ldots .]$.} & R_2EF4cyVEFd... & 09/06 1:08:44 & {$[\ldots .]$.} \\
\hline 6 & null & IN PROGRESS: $\mathbf{5 0 \%}$ & R_Q4cPvig1Xy... & completed & 33 & R_33298FbqKB... & 09/06 1:08:43 & {$[\ldots .]$.} \\
\hline 5 & null & COMPLETED & R_2zVohUheaU... & completed & 35 & R_2WVhjvzuZU... & completed & {$[\ldots .]$.} \\
\hline 4 & null & COMPLETED & R_1ONy0nNv1Z... & completed & 27 & R_2y9NR21C66 .. & completed & {$[\ldots .]$.} \\
\hline 3 & null & COMPLETED & R_2VqKMN5mnu... & completed & 50 & R_2QXULnaXwJ... & completed & {$[\ldots \ldots]$} \\
\hline 2 & null & COMPLETED & R_1GJHKOCNRG... & completed & 10 & R_2Ec3auAUWa... & completed & {$[\ldots .]$.} \\
\hline 1 & null & COMPLETED & R_221CXk8PrV... & completed & 44 & R_2SeOQn5gc1... & completed & {$[\ldots \ldots]$} \\
\hline
\end{tabular}

Figure 5. Sample screenshot of the SMARTRIQS progress monitor. Each row represents a group. Column 1 shows the group ID, column 2 shows the condition (if set), and column 3 shows the group status. Columns 4-6 show the Allocator's ID, the Allocator's time of last activity, and the Allocator's stage 1 decision (transfer to the Recipient). Columns 7-8 show the Recipient's ID and the Recipient's last activity. Column 9 is blank since Recipients do not submit any decision in the Dictator Game. 


\section{ADVANCED SETTINGS AND FEATURES}

SMARTRIQS also allows researchers to run more complex interactive studies, including but not limited to: multiple conditions within studies, group interaction up to 8 people per group, multiple stages, or repeated interaction between participants. This section briefly introduces some of the most important advanced settings of SMARTRIQS. More information, along with practical examples can be found at https://smartriqs.com/getting-started.

\section{Multiple conditions}

Researchers can set up multiple conditions within a survey by filling in the optional parameters conditions and participantCondition before the MATCH block. SMARTRIQS will then assign groups to conditions, based on these specifications, see https://smartriqs.com/randomization. Once participants are assigned to conditions, researchers can decide what should happen in each condition by using Branch Logic in the Survey Flow. For example, imagine a modified Dictator Game with three possible levels: low (10 tokens), medium (100 tokens), and high (1000 tokens), indicating that the Allocator would either allocate 10, 100, or 1000 tokens, depending on the condition. To achieve this, set conditions $=$ low, medium, high (note that there is no space between the commas and the name of the conditions) and participantCondition = random. Then, use either Branch Logic in the Survey Flow or Display Logic in the Survey Editor to determine which version of the Dictator Game is displayed to which participant. To learn more about how to implement studies such as the above example, see the "Dictator Game, 3 conditions" survey at https://smartriqs.com/demos and download the corresponding template from the OSF repository.

\section{Larger groups (3-8 participants per group)}

SMARTRIQS supports group interactions up to 8 participants per group. Researchers can assign: (a) the same role to everyone (e.g., auction, collective decision), (b) unique roles to each participant (e.g., negotiation), or (c) any combination of the above. To increase the group size, modify the value of the groupSize field to the desired number, and then add this many roles to the roles field, separating them by commas. For example, in a study with groups of four, where participants are assigned to the roles of Blue, Red, Green and Yellow, set groupSize $=4$, and roles $=$ Blue,Red,Green, Yellow (note that there is no space between role titles).

When having groups of 3-8 participants, it is also possible to set up private and group chats. While private chats are between selected participants only (excluding at least one participant), group chats include everyone in the group. Researchers can customize which participants, with whom, when, and for how long, chat in a study. As with dyadic chat, it is possible to interrupt private and group chats with unrelated tasks. In later stages, participants can also join those private chats from which they were excluded from before. To learn more about how to set up private and group chats, visit https://smartriqs.com/chat. For demos, see the "Communication" section at https://smartriqs.com/demos. The "Group Interaction" section showcases surveys with larger groups. The corresponding templates can be downloaded from the OSF repository. 


\section{Turn-taking and multiple stages}

In many studies, participants take turns or have to respond to their partner's choices. For example, the Ultimatum Game (Güth, Schmittberger, \& Schwarze, 1982) has two consecutive stages. The first stage is identical to the Dictator Game: The Allocator decides how to split a sum of money between herself and the Recipient. In the second stage, however, the Recipient can decide whether to accept or to reject the Allocator's offer. Rejecting the offer leaves both empty-handed.

To implement the above, duplicate the Dictator Game survey created in the previous section, then rename the studyID. Note that each study should have a unique studyID. Change the numStages to 2, indicating that there are two responses to be transmitted. Then, add a new question block in which the Recipient reacts to the offer, and add a new SEND block in the Survey Flow below this new question block. Finally, pipe in the Recipient's response into a new embedded data field reaction, then set sendData $=$ reaction and sendStage $=2$ (Figure S6 in the Appendix).

Next, go to the Allocator's branch, and add a new Embedded Data field named reaction under the SEND block. Also set getData $=$ Recipient, getStage $=2$, and saveData $=$ reaction, to indicate that the Recipient's reaction should be retrieved and saved into the field reaction. Then insert a new GET block under these fields. Finally, use either Branch Logic in the Survey Flow or Display Logic in the Survey Editor to display the final payoffs, conditioned on whether the offer was accepted or rejected (see Figure S7 in the Appendix).

The "Trust Game" and "Third-Party Punishment" demos at https://smartriqs.com/demos also rely on sequential interaction and multiple stages. These demos, along with the Ultimatum Game demo, were designed to help researchers learn how to implement sequential interactions and multiple stages. Corresponding survey templates are also available at the OSF repository.

\section{Simultaneous responses and waiting rooms}

In some cases researchers might want to ensure that participants start certain stages of an experiment at the exact same time (e.g., group chat, effort task). Also, if participants have to read long instructions before a task, it is likely that some of them will spend considerably more time reading instructions than others, which introduces asynchrony between participants. To ensure that participants start stages simultaneously, researchers can set up "waiting rooms" in studies.

When participants enter a waiting room, they cannot proceed to the next stage before everyone else in their group joins them in the waiting room.

To set up a waiting room, insert a SEND block and a GET block before the task that participants should start simultaneously. Set the following parameters before the SEND block: participantStatus $=$ ready, sendData $=$ participantStatus, and sendStage $=1$ (this must be a number that is not used in any other SEND block). Importantly, each waiting room counts as a separate stage - separate from other decisions - which means that researchers should not use its stage num- 
ber when sending or retrieving other responses. ${ }^{4}$ Next, set the following parameters before the GET block: getData $=$ Allocator, Recipient (the roles in the group), getStage $=1$ (this number should match the one defined above), and saveData $=$ null, null. ${ }^{5}$ Finally, set a custom message that participants will see in the waiting room. This will reduce participant concerns related to their status in the study. Setting getWaitText $=$ The task will start soon. Please wait for the other participant., for example, will display this message while participants wait to start (see Figure S8 in the Appendix).

Learn more about setting up waiting rooms by reviewing the "Effort Competition with Waiting Room" demo at https://smartriqs.com/demos and downloading the corresponding survey template from the OSF repository.

\section{Transmitting sensitive and personal data: custom private servers}

The SMARTRIQS Data Policy prohibits researchers from submitting any personal or sensitive data to the default SMARTRIQS server. Since the server is hosted at Amazon Web Services - which constitutes as a third-party - participant's confidentiality cannot be guaranteed. Researchers are allowed to submit any anonymous data (e.g., participant IDs, decisions, roles, chat logs, or open-ended text), as long as these do not contain any personal identifiers or personal addresses. Researchers who wish to use SMARTRIQS for submitting personal data or other identifiers, should set up their own server on a secure, private web-server. By using a custom server researchers can also freely modify the server-side scripts, in case they wish to modify the built-in settings of SMARTRIQS (e.g., maximum group size), or add new features to it (e.g., video chat). A step-by-step guide to setting up a custom SMARTRIQS server is available at https://smartriqs.com/custom-server.

\footnotetext{
${ }^{4}$ For example, if there is a waiting room in the Dictator Game (before the Allocator makes a decision) then the waiting room is Stage 1 and the Allocator's transfer is Stage 2. Or, if there is a waiting room in the Ultimatum Game (before the Allocator's initial offer) then the waiting room is Stage 1, the Allocator's offer is Stage 2, and the Recipient's response is Stage 3.

${ }^{5}$ Note that here the retrieved "responses" are not actual responses that we want to save. They are simply status indicators that determine whether the participant can proceed. As such, in this case we do not have to refer to any other Embedded Data when setting up the saveData parameter. Use null instead, and separate them by commas (no space between).
} 


\section{SUMMARY AND APPLICATIONS}

SMARTRIQS has a great potential for becoming a fundamental tool of social scientists, as it offers researchers the ability to run interactive studies online with unprecedented ease and efficiencywithout having to learn any programming language, installing any software, or paying for thirdparty services. Social scientists will find many potential uses of SMARTRIQS (see Table 3).

Table 3. Potential applications of SMARTRIQS across disciplines

\begin{tabular}{|c|c|}
\hline Fields / disciplines & Sample applications \\
\hline Cognitive Science & Coordination \& joint action; \\
\hline \& Linguistics & Text- and sentiment analysis in real-time communication \\
\hline Economics & $\begin{array}{l}\text { Allocation \& reciprocity (e.g., Dictator Game, Ultimatum Game); } \\
\text { Collective decision-making (e.g., Public Goods Game); } \\
\text { Hierarchical beliefs \& expectations (e.g., p-Beauty contest) }\end{array}$ \\
\hline Finance & $\begin{array}{l}\text { Simple market interactions (e.g., multiple buyers and sellers); } \\
\text { Investment decisions (e.g., Trust/Investment Game); } \\
\text { Auctions (e.g. blind, Vickrey) }\end{array}$ \\
\hline $\begin{array}{l}\text { Management \& } \\
\text { Organizational Research }\end{array}$ & $\begin{array}{l}\text { Group processes (e.g., collaboration, competition); } \\
\text { Work \& principal-agent problems (effort allocation \& provision); } \\
\text { Negotiation and strategic interactions }\end{array}$ \\
\hline $\begin{array}{l}\text { Marketing \& } \\
\text { Consumer Research }\end{array}$ & $\begin{array}{l}\text { Buyer-seller interactions (e.g., endowment effect studies); } \\
\text { Consumer focus group studies }\end{array}$ \\
\hline Political Science & $\begin{array}{l}\text { Persuasion \& spread of beliefs; } \\
\text { Political focus group studies; } \\
\text { Voting behavior }\end{array}$ \\
\hline Social Psychology & $\begin{array}{l}\text { Moral behavior (e.g., dishonesty, cheating, punishment); } \\
\text { Identity \& group dynamics (e.g., minimal group paradigms); } \\
\text { Compliance to, and enforcement of social norms; } \\
\text { Impression management \& social signaling }\end{array}$ \\
\hline $\begin{array}{l}\text { Miscellaneous } \\
\text { applications }\end{array}$ & $\begin{array}{l}\text { Educational applications (in-class experiments); } \\
\text { Field applications (field studies with participant interaction); } \\
\text { Lab studies (computerized interaction in conventional labs) }\end{array}$ \\
\hline
\end{tabular}


First and foremost, social scientists will be able to run interactive experiments online, which will allow them to recruit participants from larger and more diverse samples than when conducting conventional lab experiments. They can also easily convert their existing (non-interactive) surveys into interactive ones. Being able to match participants in real-time also makes many instances of deception unnecessary, and allows for studying human behavior in more naturalistic contexts.

Economists and researchers studying game theory can easily set up various "classic" economic games (e.g., Dictator Game, Public Goods Game), as well as novel, custom experimental designs. Researchers interested in experimental finance can conduct simple market experiments, and will be able to simulate investment decisions and auctions online.

There are plenty of potential applications in managerial and organizational contexts as well: For example, researchers can study various group processes such as collaboration and competition, task allocation, and effort provision within groups. The advanced chat feature allows scientists to study various aspects of communication: persuasion, negotiation, impression management, or even the linguistic properties of conversations, which are relevant not only in organizational research but also in political science or consumer research.

SMARTRIQS allows social psychologists to study a wide range of phenomena in online contexts, including but not limited to moral behavior, group dynamics, social norms, and impression management strategies. Psychologists interested in personality and individual differences can supplement real-time interactions with various inventories to study how personality traits and attitudes correlate with social behavior.

Importantly, the applications of SMARTRIQS are not limited to experiments conducted on Amazon Mechanical Turk or similar crowdsourcing platforms. Since SMARTRIQS has minimal technical requirements, and does not require participants to install any software - any device with an Internet access and a browser that supports HTML5 and JavaScript suffices - researchers can recruit participants in virtually any context: in conventional computer labs, in classrooms, or even in the field. This makes it possible to conduct studies with real-time interaction in places where it would have been challenging before (e.g., developing countries, remote locations, events). SMARTRIQS can also be used for educational purposes: Teachers can set up simple interactive experiments, then have their students complete these studies in class. With the built-in ResultsReports function of Qualtrics, teachers can even display the results to students in real-time.

SMARTRIQS can also be combined with other useful tools developed for Qualtrics. For example, researchers who study dynamic cognitive processes can supplement SMARTRIQS surveys with a tool that captures mouse cursor trajectories (Mathur \& Reichling, 2019). Similarly, researchers who want to measure how much time participants spend on-screen versus off-screen (a crucial metric of participant attention and engagement) can supplement SMARTRIQS with TaskMaster, a simple tool that tracks participants' activity (Permut, Fisher, \& Oppenheimer, 2019).

Finally, since SMARTRIQS is free, open-source, and provides a standardized generic framework for interactive studies, it will not only serve as a useful experimental tool for psychologists but also contribute to, and propagate, open research practices in psychology. 
Acknowledgements. I thank Ankita Sastry for her help in developing SMARTRIQS and Peggy He, Jinny Hwang, Denise Lin, and Sommer Schneller for their assistance in testing SMARTRIQS. I also thank Russell Golman, Nik Gurney, Einav Hart, Mark Hurlstone, Daisung Jang, Melis Kirgil, Jonathan Lee, Stephanie Permut, Eric VanEpps, Chao Wang, and Simon van der Zandt for their valuable feedback and suggestions.

\section{Important Links}

1. Live demos: https://smartriqs.com/demo

2. SMARTRIQS registration form: LINK

3. Survey templates (QSF files): https://osf.io/cgejr

4. Data collection progress monitor: https://server.smartriqs.com/php/monitor.html

5. Data Policy \& Data Submission Policy Agreement: https://smartriqs.com/data-policy

6. Source code (JavaScript and PHP): https://github.com/andras-molnar/smartriqs

\section{REFERENCES}

Arechar, A. A., Gächter, S., \& Molleman, L. (2018). Conducting interactive experiments online. Experimental Economics, 21(1), 99-131. doi: 10.1007/s10683-017-9527-2

Balietti, S. (2017). nodeGame: Real-time, synchronous, online experiments in the browser. Behavior Research Methods, 49(5), 1696-1715. doi: 10.3758/s13428-016-0824-z

Berinsky, A. J., Huber, G. A., \& Lenz, G. S. (2012). Evaluating Online Labor Markets for Experimental Research: Amazon.com's Mechanical Turk. Political Analysis, 20(3), 351-368. doi: $10.1093 / \mathrm{pan} / \mathrm{mpr} 057$

Bohannon, J. (2016). Mechanical Turk upends social sciences. Science, 352(6291), 1263-1264. doi: 10.1126 /science.352.6291.1263

Casari, M., \& Cason, T. N. (2009). The strategy method lowers measured trustworthy behavior. Economics Letters, 103(3), 157-159. doi: 10.1016/j.econlet.2009.03.012

Chandler, J., Rosenzweig, C., Moss, A. J., Robinson, J., \& Litman, L. (2019). Online panels in social science research: Expanding sampling methods beyond Mechanical Turk. Behavior Research Methods, 51 (5), 2022-2038. doi: 10.3758/s13428-019-01273-7

Chen, D. L., Schonger, M., \& Wickens, C. (2016). oTree-An open-source platform for laboratory, online, and field experiments. Journal of Behavioral and Experimental Finance, 9, 88-97. doi: $10.1016 /$ j.jbef.2015.12.001

Collaboration*, O. S. (2015). Estimating the reproducibility of psychological science. Science, 349(6251), aac4716-aac4716. doi: 10.1126/science.aac4716

Fischbacher, U. (2007). z-Tree: Zurich toolbox for ready-made economic experiments. Experimental Economics, 10(2), 171-178. doi: 10.1007/s10683-006-9159-4

Forsythe, R., Horowitz, J. L., Savin, N., \& Sefton, M. (1994). Fairness in Simple Bargaining Experiments. Games and Economic Behavior, 6(3), 347-369. doi: 10.1006/game.1994.1021 
Giamattei, M., Molleman, L., Yahosseini, K., \& Gächter, S. (2019). LIONESS Lab - a free webbased platform for conducting interactive experiments online.

Gosling, S. D., \& Mason, W. (2015). Internet Research in Psychology. Annual Review of Psychology, 66(1), 877-902. doi: 10.1146/annurev-psych-010814-015321

Güth, W., Schmittberger, R., \& Schwarze, B. (1982). An experimental analysis of ultimatum bargaining. Journal of Economic Behavior \& Organization, 3(4), 367-388. doi: 10.1016/ 0167-2681(82)90011-7

Hawkins, R. X. D. (2015). Conducting real-time multiplayer experiments on the web. Behavior Research Methods, 47(4), 966-976. doi: 10.3758/s13428-014-0515-6

Hendriks, A. (2012). SoPHIE - Software Platform for Human Interaction Experiments.

Henninger, F., Kieslich, P. J., \& Hilbig, B. E. (2017). Psynteract: A flexible, cross-platform, open framework for interactive experiments. Behavior Research Methods, 49(5), 1605-1614. doi: 10.3758/s13428-016-0801-6

Henrich, J., Heine, S. J., \& Norenzayan, A. (2010). The weirdest people in the world? Behavioral and Brain Sciences, 33(2-3), 61-83. doi: 10.1017/S0140525X0999152X

Hertwig, R., \& Ortmann, A. (2008). Deception in Experiments: Revisiting the Arguments in Its Defense. Ethics \& Behavior, 18(1), 59-92. doi: 10.1080/10508420701712990

Mao, A., Chen, Y., Parkes, D. C., \& Procaccia, A. D. (2012). TurkServer: enabling synchronous and longitudinal online experiments. Human Computation(March), 33-39.

Mason, W., \& Suri, S. (2012). Conducting behavioral research on Amazon's Mechanical Turk. Behavior Research Methods, 44(1), 1-23. doi: 10.3758/s13428-011-0124-6

Mathur, M. B., \& Reichling, D. B. (2019). Open-source software for mouse-tracking in Qualtrics to measure category competition. Behavior Research Methods. doi: 10.3758/s13428-019-01258 $-6$

Molnar, A. (2019). SMARTRIQS: A Simple Method Allowing Real-Time Respondent Interaction in Qualtrics Surveys. Journal of Behavioral and Experimental Finance, 22, 161-169. doi: 10.1016/j.jbef.2019.03.005

Paolacci, G., \& Chandler, J. (2014). Inside the Turk. Current Directions in Psychological Science, 23(3), 184-188. doi: 10.1177/0963721414531598

Peer, E., Brandimarte, L., Samat, S., \& Acquisti, A. (2017). Beyond the Turk: Alternative platforms for crowdsourcing behavioral research. Journal of Experimental Social Psychology, 70 (January), 153-163. doi: 10.1016/j.jesp.2017.01.006

Permut, S., Fisher, M., \& Oppenheimer, D. M. (2019). TaskMaster: A Tool for Determining When Subjects Are on Task. Advances in Methods and Practices in Psychological Science, 2(2), 188-196. doi: 10.1177/2515245919838479

Pettit, J., Friedman, D., Kephart, C., \& Oprea, R. (2014). Software for continuous game experiments. Experimental Economics, 17(4), 631-648. doi: 10.1007/s10683-013-9387-3

Qualtrics. (2020). Provo, Utah, USA: Qualtrics. Retrieved from https://www .qualtrics.com

SurveyMonkey. (2020). San Mateo, California, USA: SurveyMonkey Inc. Retrieved from www .surveymonkey.com

Zhou, H., \& Fishbach, A. (2016). The pitfall of experimenting on the web: How unattended selective attrition leads to surprising (yet false) research conclusions. Journal of Personality and Social Psychology, 111(4), 493-504. doi: 10.1037/pspa0000056 


\section{APPENDIX}

Change Question Type

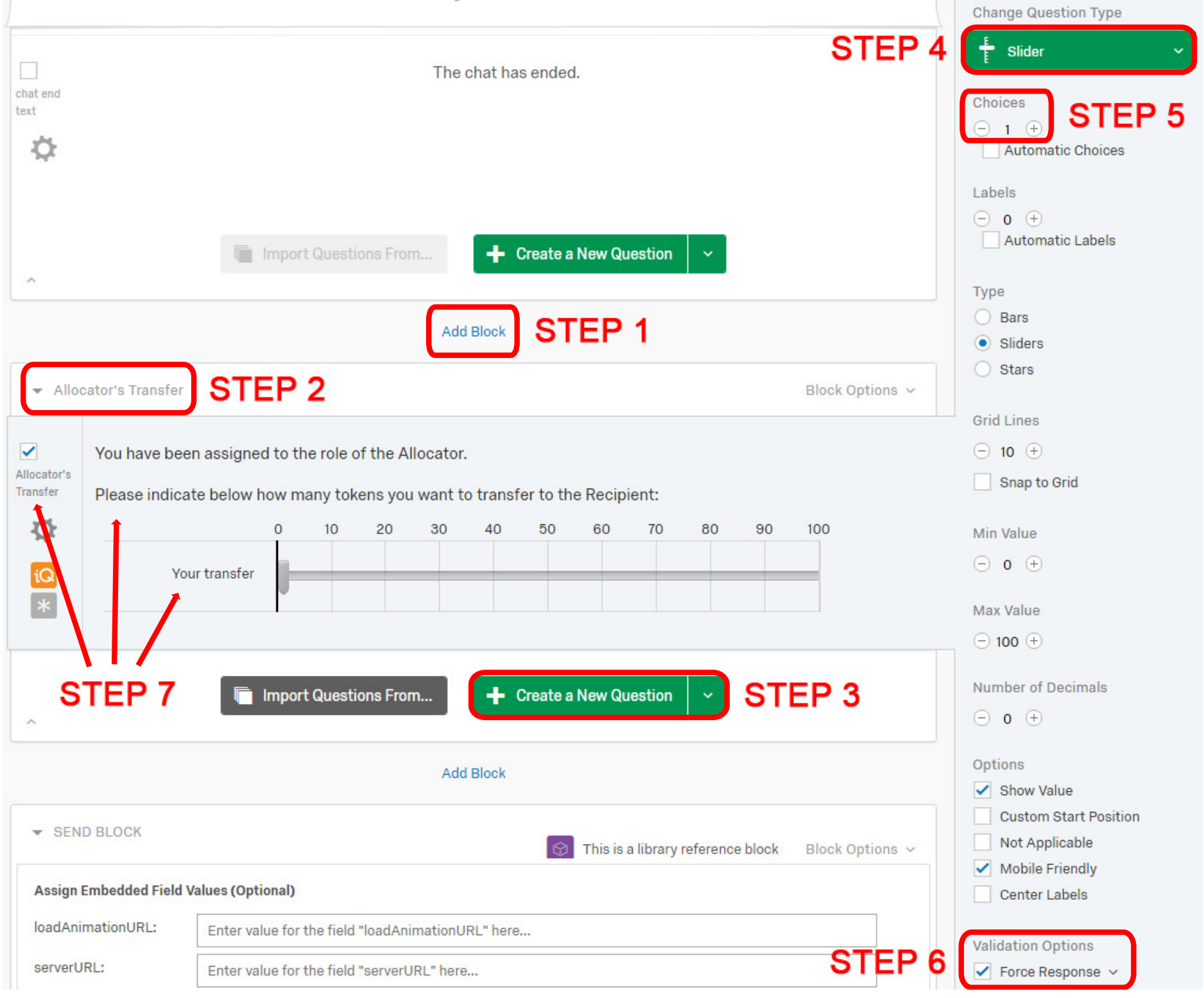

Figure S1. Adding a new block and question in the Qualtrics Survey Editor. The example above shows the setup of a question that records the Allocator's transfer in the Dictator Game (slider scale from 0 to 100). To change the default value (0), drag the slider to the desired position. 


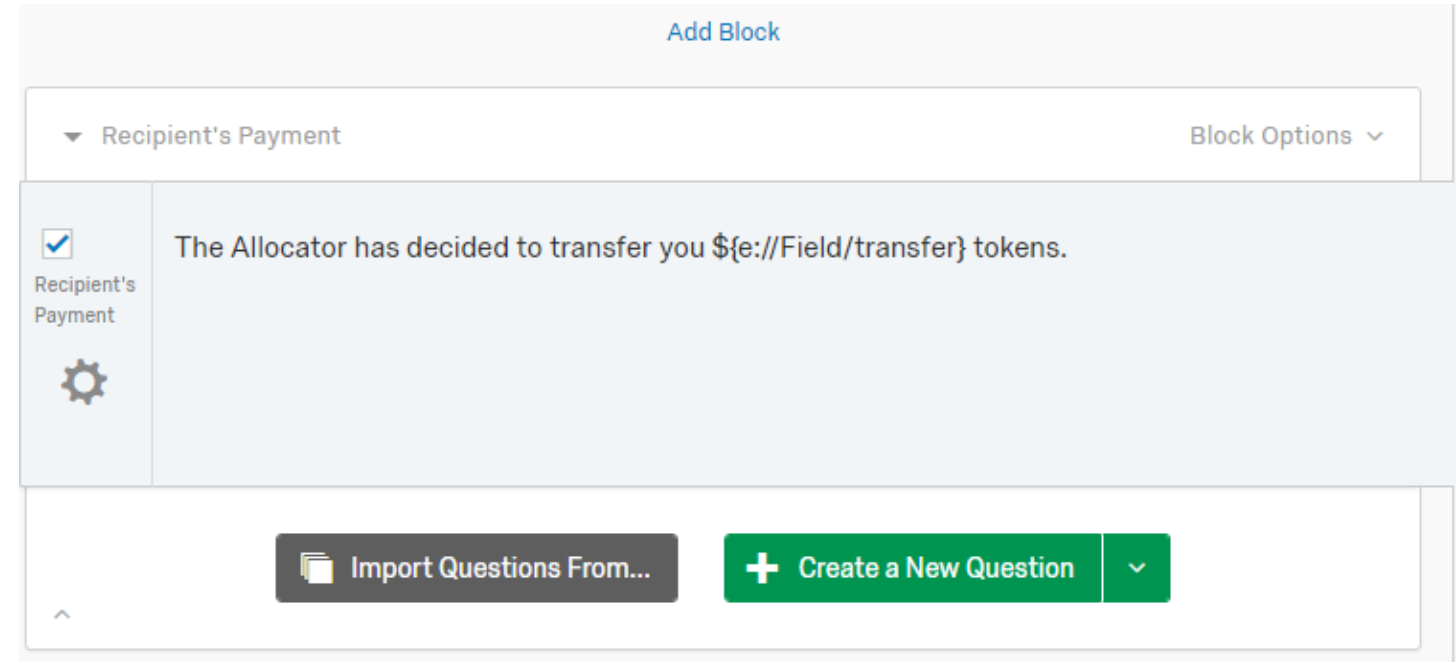

\section{$\boldsymbol{A}$ Text/Graphic}

Choices

$\odot 0 \oplus$

Automatic Choices

Type

- Text

Graphic

File

Validation Type

- None

Custom Validation

Figure S2. Sample survey question (Recipient's payment) 


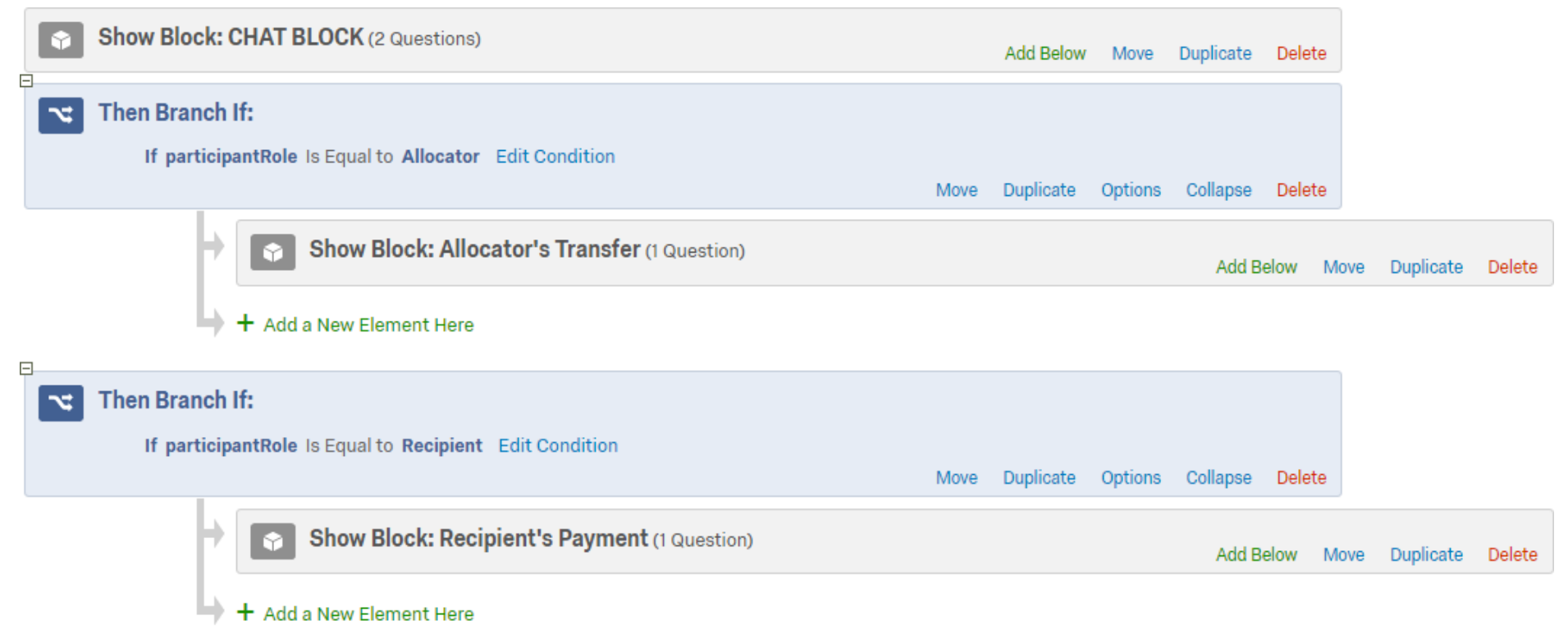

Figure S3. Using Branch Logic to conditionally display blocks to participants. In this example we use the participantRole variable as the condition. Depending on the value of this variable (i.e, the participant's role), we display either only the transfer block or only the payment block. 


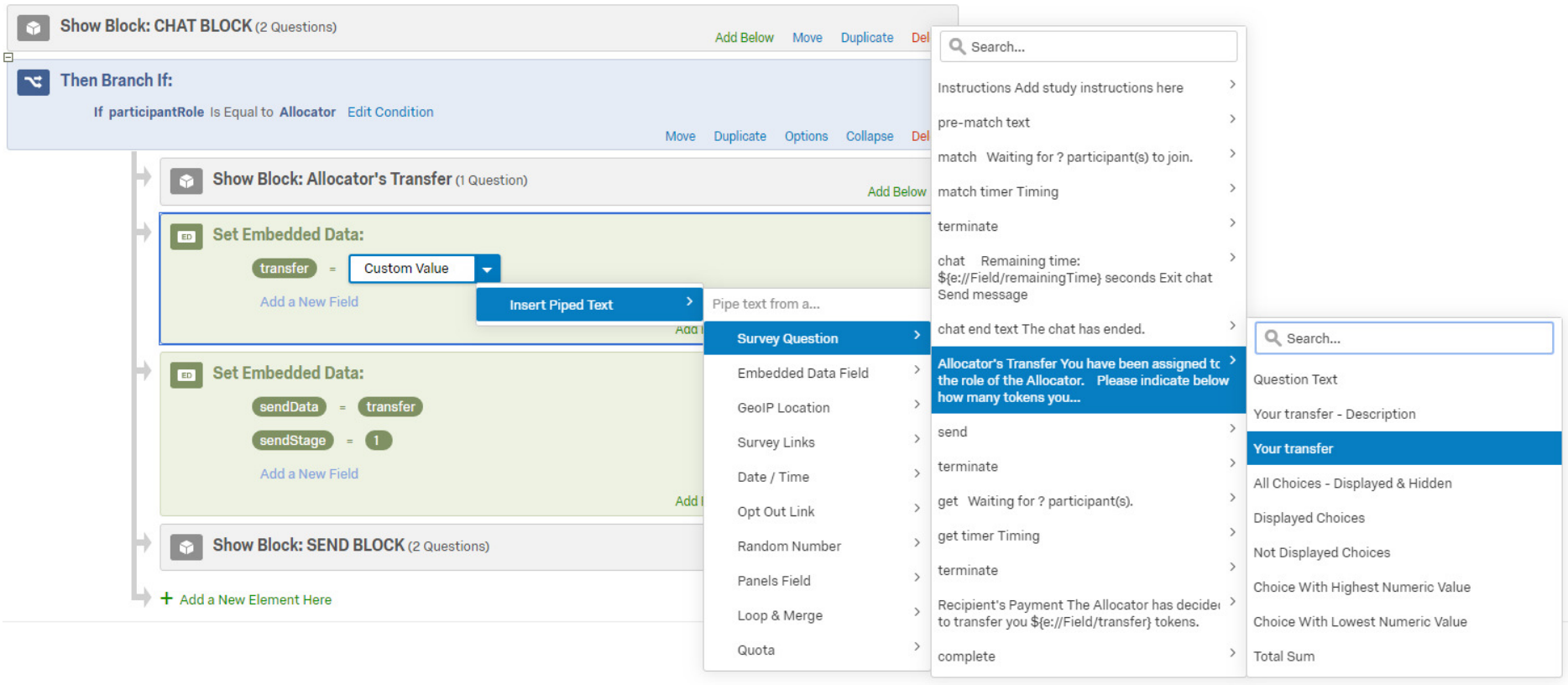

Figure S4. Setting up a SEND block: Inserting a response as Piped Text. 


\section{A End of Survey Move Duplicate $\sim$ Customize Delete}

Figure S5. The COMPLETE block and an optional End of Survey Element. The COMPLETE block should always be the last block in SMARTRIQS surveys, unless, if there is a custom End of Survey Element. In that case (as in the example above), the End of Survey Element should be placed after the COMPLETE block. 
$\checkmark$ Then Branch If:

If participantRole Is Equal to Recipient Edit Condition

Move Duplicate Options Collapse Delete

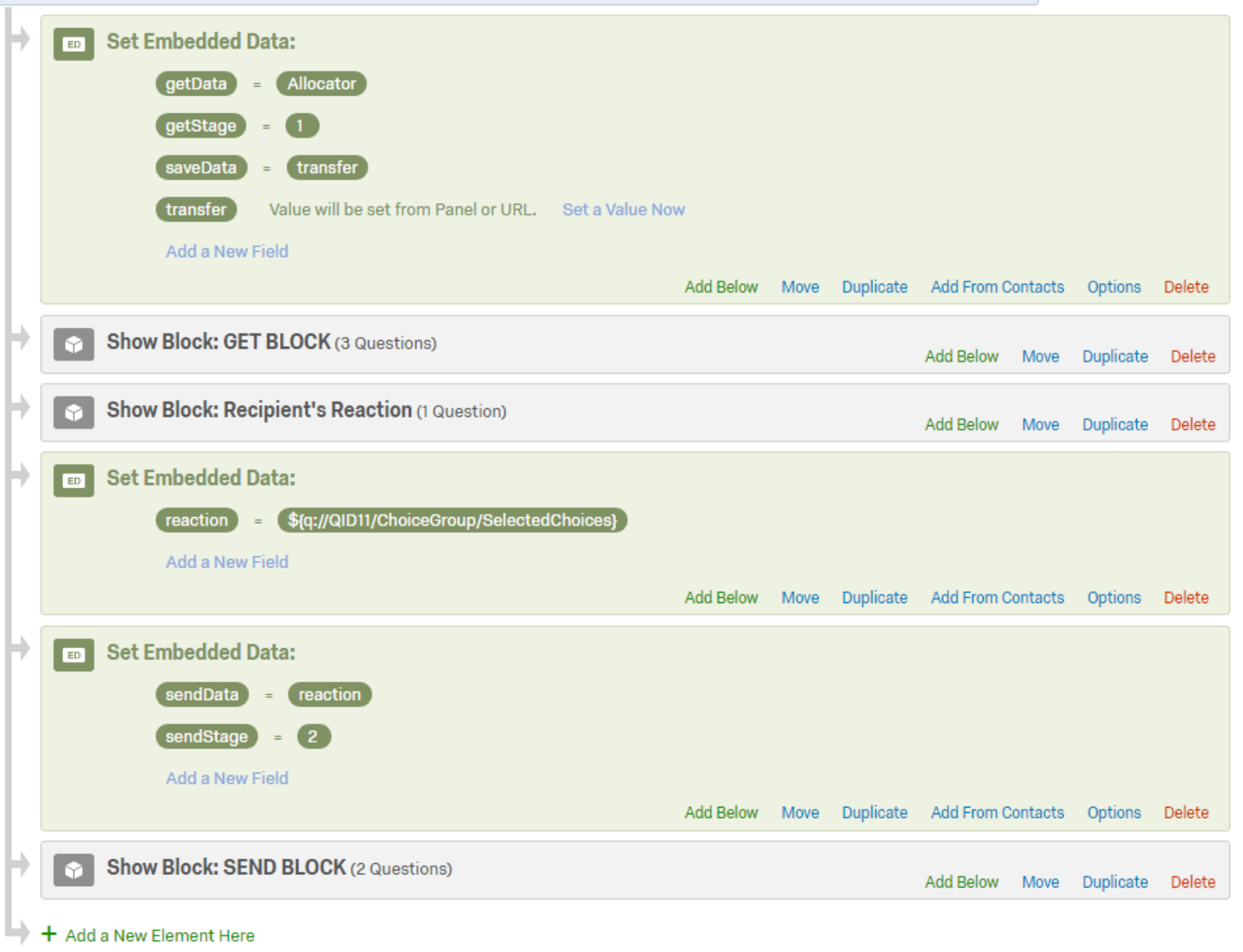

Figure S6. Sample setup of the Recipient's branch in an Ultimatum Game. First, the Allocator's offer is retrieved via a GET block, then the Recipient's reaction is transmitted via a SEND block. The new panels below the "Recipient's Reaction" block were added by clicking on the "Add a New Element Here" button, and then either selecting "Embedded Data" or "Block." 


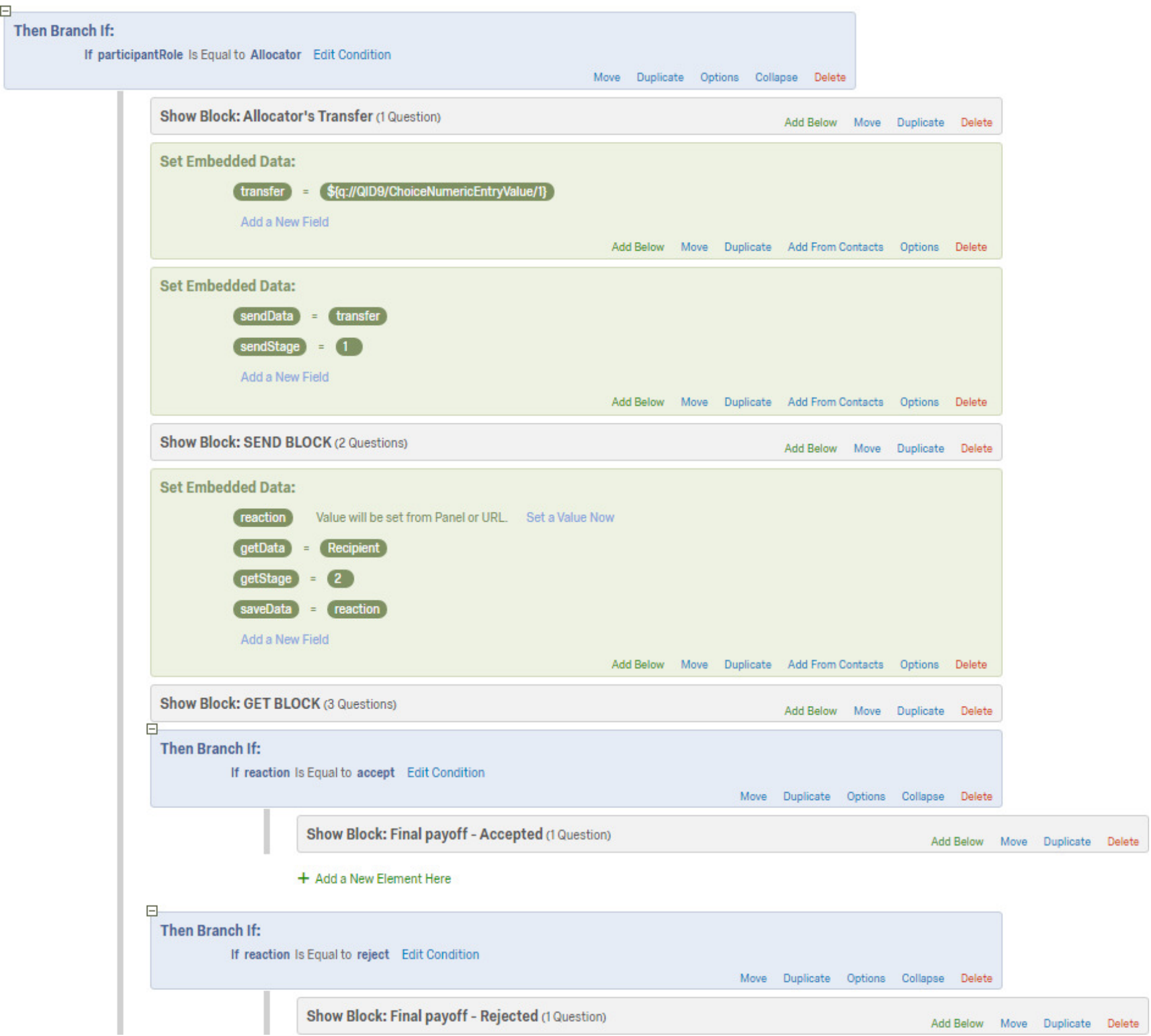

Figure S\%. Sample setup of the Allocator's branch in an Ultimatum Game. First, the Allocator's offer is transmitted via a SEND block, then the Recipient's reaction is retrieved via a GET block. The branches below the GET block use the retrieved response to display either the "Accepted" or the "Rejected" blocks. 


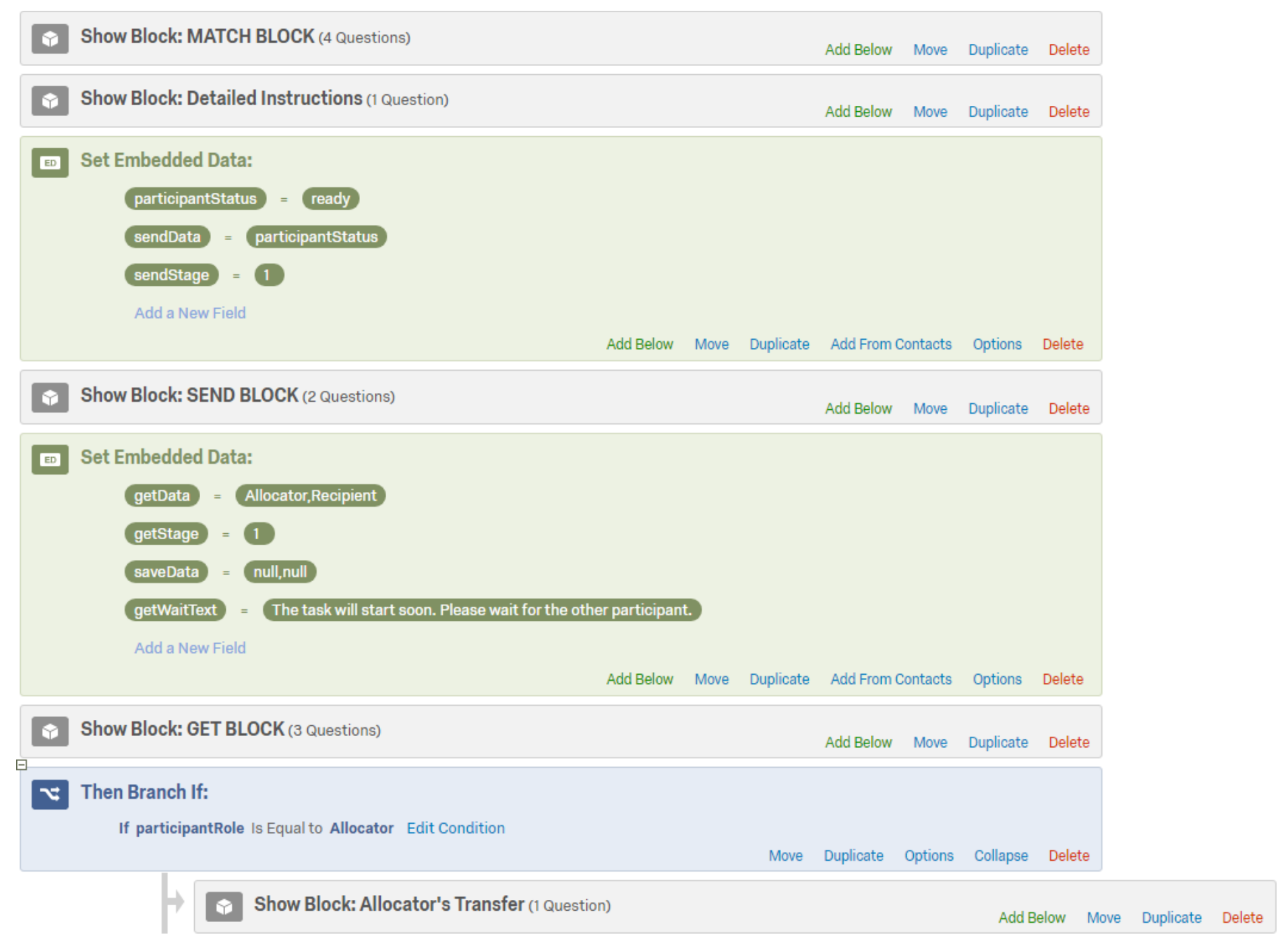

Figure S8. Sample setup of a waiting room. Here the waiting room is inserted after the instructions, but before the Allocator's branch. Both participants have to read the instructions first and proceed to the waiting room. Note that a waiting room counts as a separate stage, so in this example the Allocator's transfer would be already Stage 2 . 